\title{
Lipödem - Mythen und Fakten Teil 3
}

\author{
T. Bertsch ${ }^{1} ;$ G. Erbacher ${ }^{1,2}$ \\ ${ }^{1}$ Földiklinik Hinterzarten - Europäisches Zentrum für Lymphologie; ${ }^{2}$ Dipl.-Psychologin, Psychologische Psychothera- \\ peutin, Supervisorin (hsi)
}

\section{Schlüsselwörter}

Lipödem, Adipositas, Gewichtsabnahme, Wissenschaftliche Evidenz

\section{Zusammenfassung}

Um das Lipödem ranken sich zahlreiche Mythen! In dieser kleinen Übersichtsreihe über die Mythen des Lipödems werfen wir einen kritischen Blick auf populäre Statements zum Lipödem; Statements, die vor Jahrzehnten schon Eingang in wissenschaftliche Publikationen gefunden haben und seither unkritisch und stetig wiederholt werden; Statements, die dadurch inzwischen zum selbstverständlichen Wissensallgemeingut von Lipödempatientinnen und vor allem auch von LipödemSelbsthilfegruppen geworden sind. Im ersten Teil unserer Darstellung haben wir uns kritisch mit zwei populären Mythen über das Lipödem auseinandergesetzt. Hierbei haben wir festgestellt, dass sowohl für das Statement "Das Lipödem ist eine progrediente Erkrankung" als auch für das Statement "Ein Lipödem macht psychisch krank" keine wissenschaftliche Evidenz vorliegt. In einem zweiten Beitrag über die Mythen des Lipödems fokussierten wir uns auf den Ödemaspekt, auf das „Ödem im Lipödem" und die hieraus erfolgende therapeutische Konsequenz: die Manuelle Lymphdrainage. Wir konnten darlegen, dass für das populäre Statement "Das Lipödem ist in erster Linie ein "Ödem-Problem", daher ist die Manuelle Lymphdrainage essenzielle und regelmäßig durchzuführende Standardtherapie" ebenfalls keine wissenschaftliche Evidenz existiert. Der regelmäßigen und dauerhaften Verord- nung von Manuellen Lymphdrainagen mit dem Ziel der "Ödembeseitigung" fehlt daher jede Grundlage. In diesem dritten Teil der Auseinandersetzung über bekannte und oft zitierte" Lipödem-Statements" beschäftigen wir uns mit zwei weiteren Mythen: 4. "Das Lipödem macht dick" und 5. "Gewicht abnehmen hat keinen Effekt auf das Lipödem". Für beide Statements gibt es weder ein sinnvolles physiologisches bzw. pathophysiologisches Konstrukt noch eine sich in der Literatur findende wissenschaftliche Evidenz. Darüber hinaus widersprechen beide Statements in hohem Maße unserer seit Jahren bestehenden täglichen klinischen Erfahrung mit Lipödempatientinnen. Tatsächlich scheint das Gegenteil richtig: Gewichtszunahme wirkt als entscheidender Trigger, um - bei entsprechend genetischer Disposition für ein Lipödem - dieses überhaupt erst zu entwickeln. Lipödem und Adipositas sind zwei unterschiedliche Erkrankungen, die jedoch in den meisten Fällen gemeinsam auftreten. Fast täglich sehen wir Lipödempatientinnen, die sich aufgrund ihrer morbiden Adipositas einer bariatrischen Operation unterzogen und dadurch effektiv - auch im Bereich der Extremitäten - Gewicht verloren haben. Patientinnen mit Lipödem erfahren durch diese meist nachhaltige Gewichtsabnahme regelhaft eine deutliche Besserung ihrer lipödemtypischen Schmerzen. Häufig sind die Patienten dann beschwerdefrei, sodass wir dann von einem Lipödem in Remission sprechen können. In einem vierten Beitrag werden wir uns mit dem Stellenwert der Liposuktion beim Lipödem beschäftigen, um dann in unserer letzten Dar-

Lipoedema - myths and facts Part 3

Phlebologie 2018; 47: 188-197

https://doi.org/10.12687/phleb2421-4-2018

Eingereicht: 02. Mai 2018

Angenommen: 14. Mai 2018

English version available at: www.thieme.de/phlebo stellung ein therapeutisches Konzept vorzustellen, das nicht nur wissenschaftlich fundiert ist, sondern auch zu einer nachhaltigeren und umfassenderen Beschwerdebesserung unserer Lipödempatientinnen beitragen soll.

\section{Keywords}

Lipedema, Lipoedema, obesity, weight loss, scientific evidence

\section{Summary}

Lipedema is associated with numerous myths. In this short series we offer an overview of the myths of lipedema, and we throw a critical eye over popular statements concerning the disease; statements that found their way into scientific publications decades ago, and which have been accepted and repeated since then without criticism; statements which have become widely accepted facts for lipedema patients, and particularly for lipedema self-help groups. In the first part of this series we took a critical look at two popular myths about lipedema. We found that there is no scientific evidence for neither "Lipedema is a progressive disease", nor "Lipedema negatively affects mental health". In the second article about the myths of lipedema we focussed on the edema aspect, on the "edema in lipedema" and the subsequent therapeutic consequences - manual lymph drainage. We were also able to show that there is no scientific evidence for the popular statement: "lipedema is primarily an "edema problem"; manual lymph drainage is thus the essential standard therapy to be conducted regularly". There is thus no basis for the prescription of long term regular manual lymph drainage with the aim of removing edema. In this, the third part of the series on familiar and often quoted "lipedema statements" we take a closer look at two other myths: 4: "lipedema makes you fat" and 5: "weight loss 
has no effect on lipedema". For both statements there is neither a reasonable physiological or pathophysiological construct nor is there any scientific evidence in the literature. Furthermore both statements contradict our many years of daily clinical experience with lipedema patients to a high degree. It actually seems that the converse is true: Weight gain seems to be a decisive trigger for the development of lipedema, in patients with the right genetic disposition. Lipedema and obesity are two different diseases, but mostly they appear together. We see patients on almost a daily basis, who effectively lose weight after successful bariatric surgery as part of our obesity program, including in the limbs. Lipedema patients regularly experience considerable improvement in the pain symptoms typical for lipedema through sustained weight loss. These patients are often symp- tom-free, so that we can then say that the lipedema is in remission. In our fourth contribution to the series we will examine the significance of liposuction for lipedema, in order to then present a therapeutic concept in the last part of the series, that is not only based on scientific evidence, but should also contribute to a long-term and comprehensive improvement in the symptoms of lipedema patients.

\section{Einleitung}

Dies ist bereits der dritte Teil einer Darstellung, die sich kritisch mit populären Lipödem-Statements beschäftigt, Mythen, die sich seit Jahrzehnten um die Erkrankung Lipödem ranken.

Im ersten Teil unserer Reihe über das Lipödem stellten wir fest, dass für die überall zu lesende Behauptung: „Das Lipödem ist eine progrediente Erkrankung" keine wissenschaftliche Evidenz vorliegt; der oft missbrauchte Terminus „Lipolymphödem“ ist daher obsolet (1). Nicht die Erkrankung Lipödem ist progredient, häufig allerdings die Erkrankung Adipositas. Liegen daher sowohl ein Lipödem als auch ein Lymphödem bei der schwer adipösen Patientin vor, handelt es sich somit nicht um ein „Lipolymphödem“, sondern in der Regel um ein Adipositas-assoziiertes Lymphödem (2).

Darüber hinaus konnten wir im Rahmen unserer Pilotstudie zeigen, dass auch das Statement „Das Lipödem macht psychisch krank" ein Mythos ist, dem jede wissenschaftliche Grundlage fehlt. Vielmehr scheint eine hohe psychische Vulnerabilität ganz wesentlich zur Entwicklung eines Lipödems beizutragen - nicht zuletzt auch aufgrund der gesteigerten Schmerzwahrnehmung (3).

Im zweiten Teil unserer Übersichtsarbeit widmeten wir uns dem Statement „Das Lipödem ist in erster Linie ein „Ödem-Problem“, daher ist die Manuelle Lymphdrainage essenzielle und regelmäßig durchzuführende Standardtherapie“. Wir konnten zeigen, dass weder klinische, bildgebende noch histologische Hinweise für ein „Ödem im Lipödem“ existieren. Folglich fehlt auch die Indikation für dauerhaf- te und regelmäßig durchzuführende Manuelle Lymphdrainagen (4).

Dieser dritte Teil unserer Darstellung über die Mythen des Lipödems beschäftigt sich mit dem heiklen Thema „Lipödem und Adipositas“. Hier sind es vor allem zwei Statements, die uns - täglich - in unserer klinischen Arbeit mit Lipödempatientinnen begegnen. Einmal die vor allem bei Patientinnen populäre Feststellung: „Das Lipödem macht dick!“ und zum anderen die von vielen Lipödemexperten verbreitete Botschaft“ „Gewicht abnehmen hat keinen Effekt auf das Lipödem!“

Wir weisen gleich zu Beginn unserer Ausführungen darauf hin, dass für beide Mythen keine wissenschaftliche Datenlage existiert. Für die Gegenthese, also für die Aussage, dass ein Lipödem nicht zur Gewichtszunahme führt und dass Gewichtsabnahme durchaus eine Verbesserung der Beschwerden zur Folge hat, allerdings auch nicht!

Gleichwohl erscheint es offensichtlich, dass ein enger Zusammenhang zwischen Übergewicht bzw. Adipositas und dem Krankheitsbild Lipödem vorliegt. In unserer kassenärztlichen lymphologischen Ambulanz sahen wir 20152344 Patientinnen mit der Diagnose Lipödem. Nur $3 \%$ dieser Patientinnen waren normalgewichtig, $9 \%$ übergewichtig (BMI zwischen 25 und $30 \mathrm{~kg}$ $\mathrm{m} /{ }^{2}$ ) und $88 \%$ unserer Lipödempatientinnen waren adipös $\left(\right.$ BMI $\left.>30 \mathrm{~kg} / \mathrm{m}^{2}\right)$ (5). Unser Patientengut scheint hier weitgehend repräsentativ zu sein. So beschreibt Bosman in einem niederländischen Zentrum bis zu 80 \% ihrer Lipödempatientinnen als übergewichtig und/oder adipös (nicht aufgeschlüsselt in Übergewicht und Adipositas) (6). Die britische Arbeitsgruppe um Child and Gordon publizierte be- reits 2011, dass nur 4 \% ihrer Lipödempatientinnen normalgewichtig sind, $11 \%$ übergewichtig und $85 \%$ adipös (7). Beim Betrachten dieser Zahlen wird der starke Zusammenhang zwischen Lipödem und Adipositas deutlich. Die normalgewichtige Lipödempatientin ist eine Rarität!

In diesem Kontext muss berücksichtigt werden, dass der BMI bei Lipödempatientinnen im Übergewichtsbereich (zwischen 25 und $30 \mathrm{~kg} / \mathrm{m}^{2}$ ) nur eingeschränkt verwertbar ist. Es gibt die seltene Patientengruppe mit weitgehend schlankem Oberkörper und deutlicher Fettgewebsvermehrung der Extremitäten. Hier wird „formal“ Übergewicht vorgetäuscht, die durch die Fettgewebsverteilung aufgrund der Lipohypertrophie der Beine tatsächlich nicht gegeben ist. Für diese Patienten ist der WHtR (Waist-to-Height Ratio, Taille-zuGröße-Verhältnis') das geeignetere Maß. Der WHtR bezeichnet das Verhältnis zwischen Taillenumfang und Körpergröße und gibt eine bessere Aussage zur Verteilung des Körperfettes. In der Földiklinik wird bei allen Patienten mit Lipödem sowohl der BMI als auch der WHtR ermittelt.

Wie ist nun aber dieser offensichtliche Zusammenhang zwischen Lipödem und Adipositas zu erklären?

In Ermangelung einer wissenschaftlichen Datenlage wollen wir beide populären Statements auf Plausibilität und empirische Evidenz untersuchen.

\section{Mythos 4: Das Lipödem macht dick!}

Von Patientenseite aber auch von Medien wird der Zusammenhang zwischen Lipödem und Adipositas gerne damit erklärt, 
dass das Lipödem für die Gewichtszunahme der Lipödempatientin verantwortlich ist. So schreibt das Magazin Stern: „Frauen, die unter einem Lipödem leiden, werden meist fälschlicherweise für schlicht übergewichtig gehalten“... und weiter... „viele betroffene Frauen (und wenige Männer) sind ihr Leben lang schlank gewesen, bevor die Fettpolster an Beinen und Armen plötzlich unkontrolliert anwachsen" (8).

Sputnik, das Jugendradio des Mitteldeutschen Rundfunks, beschreibt auf seiner Website das Lipödem als „mysteriöse Krankheit, die dick macht" (9).

Bei Evidero, einem Internet-Gesundheitsportal, ist das Lipödem als erste Krankheit aufgeführt, die zu Übergewicht führen kann, noch vor der Hypothyreose. Dort liest man: „Ein Lipödem hat nichts mit der Nahrungsaufnahme zu tu“" (10).

Während der Vorbereitung auf diesen Artikel wird im NDR-Fernsehen ein Beitrag zum Thema Lipödem in der Sendung "Markt" gesendet. Hier wird gleich zu Beginn des Einspielers eine schwer adipöse Patientin vorgestellt, die mühsam eine Treppe hochgeht. Gleichzeitig hört man die Stimme aus dem „off“: „Treppensteigen, für R. W. eine schmerzhafte Tortur. Sie leidet an einer seltenen Krankheit: Lipödem, eine Fettverteilungsstörung, durch die nimmt sie immer mehr zu" (11, statt des Patientennamens hier nur die Initialen).

Auf Youtube wird von einer schwer adipösen Lipödemaktivistin inzwischen sogar das „Lipödem im Bauch“ propagiert (12).

Noch verbreiteter ist diese Betrachtungsweise in den USA. Dort gibt es eine Koalition aus Ärzten und Patientinnen, die Gewichtszunahme mit der Krankheit Lipödem zu erklären. Im Rahmen eines Fachartikels von Dr. Karen Herbst, Endokrinologin und Protagonistin der amerikanischen Lipödemszene, schreibt eine Patientin, deren Gewicht bei über 200 kg liegt:

„I have a congenital condition called lipedema that causes my body to produce excess adipose tissue, also known as fat" (13). In einem US-Internetportal für übergewichtige Menschen liest man: „Lipedema May Increase Obesity in Women" (14).

Folge dieser in den Medien propagierten Sichtweise sind Patientinnen mit der Diagnose Lipödem, die uns täglich in der Fachklinik für Lymphologie aber auch in der kassenärztlichen lymphologischen Ambulanz mit diesen Sätzen konfrontieren: „Meine Beine werden immer dicker und tun weh". Wohlgemerkt: Die diesen Satz aussprechende Patientin wiegt z. B. 90 kg oder z. B. 140 kg. Der Fokus der Patientin liegt aber explizit auf den Beinen. Dem folgt dann häufig das zweite Statement: „Ich nehme wegen meines Lipödems stetig Gewicht zu“.

Eine wissenschaftliche Evidenz für diese Betrachtungsweise gibt es nicht! Auch fehlt ein schlüssiges pathophysiologisches Konstrukt, welches erklärt, auf welche Weise ein Lipödem zu Gewichtszunahme führt, zu Gewichtszunahmen von 20 kg, 50 kg oder $70 \mathrm{~kg}$ !

Allgemeiner Konsens besteht darin, dass eine disproportionale Fettgewebsvermehrung der Beine (manchmal auch der Arme) ein wesentliches Kriterium für diese Erkrankung darstellt. Diese initial oft nur diskrete Disproportion zeigt sich häufig bereits während der Pubertät. Beschwerden haben die jungen Frauen in dieser Zeit üblicherweise noch nicht; es besteht somit allenfalls eine milde Lipohypertrophie der Beine, die keinerlei Krankheitswert hat. Erst durch die Gewichtsprogredienz und die damit einhergehende überproportionale Fettgewebszunahme der unteren Körperhälfte steigt das Risiko für Beschwerden an - und damit auch für die Entwicklung eines Lipödems.

Vieles spricht daher für die gegenteilige Position: Nicht das Lipödem führt zur Gewichtszunahme, sondern Gewichtszunahme (häufig Adipositas) kann bei entsprechender (genetischer) Disposition ein Lipödem zur Folge haben.

Die Ursachen von Adipositas sind vielfältig und sehr komplex. Wissenschaftlich evident sind genetische und vor allem auch epigenetische Einflüsse (15-18). Biologische Faktoren wie Stress oder Suchtverhalten können das Gewicht beeinflussen (19-20). Psychische Erkrankungen wie Depressionen und Essstörungen tragen stark zur Adipositas bei (21). Schließlich spielen auch soziokulturelle Veränderungen eine erhebliche Rolle bei der Entwicklung von Adipositas: beispielsweise Veränderungen des Essverhaltens, der exorbitante Zuckerkonsum (vor allem in Soft Drinks und industriell hergestelltem Essen), ein bewe- gungsarmer Lifestyle sowie ein Schönheitsideal, das im Untergewichtsbereich angesiedelt ist - und daran eng gekoppelt eine Diätkultur, die oft bereits im Jugendalter beginnt und im Langfristverlauf nicht zur Gewichtsabnahme sondern im Gegenteil zu Gewichtszunahme führt (22-26).

In Deutschland sind inzwischen knapp $25 \%$ der Menschen adipös (BMI > 30 $\mathrm{kg} / \mathrm{m}^{2}$ ), in den USA waren es 2017 bereits $40 \%(27-28)$.

Wie eine Epidemie hat sich Adipositas in den vergangenen Jahrzehnten verbreitet (29) und - in Folge dessen - auch die Diagnose Lipödem!

Wenn das Lipödem aber nicht dick macht, wie erklärt sich dann die dennoch breite Unterstützung für diese wissenschaftlich unhaltbare Position?

Wesentliche Grundlage für die Popularität dieser Sicht auf das Lipödem ist die Stigmatisierung der Krankheit Adipositas. Adipositas wird vielfach als Ergebnis schuldhaften Verhaltens gewertet, als Resultat von Willensschwäche oder Disziplinlosigkeit. Gerade unter medizinischem Fachpersonal und hier besonders unter Ärzten ist gewichtsbezogene Diskriminierung und Stigmatisierung besonders ausgeprägt (30-32). Schon Medizinstudenten empfinden herabwertende Witze über adipöse Menschen nicht als unangebracht, da diese - so die Haltung - selbst für das Übergewicht verantwortlich seien. Aber auch bei auf Adipositas spezialisierten Ärzten und bei Diätassistenten konnten stigmatisierende Einstellungen festgestellt werden (33-34).

Die ärztliche Empfehlung zur Gewichtsreduktion findet sich regelhaft in Arztbriefen der uns zugewiesenen Patientinnen. Wie oben skizziert, sind die Ursachen der auch von der WHO anerkannten - Krankheit Adipositas komplex. „Schuld“ (bzw. "der Vorwurf des Fehlverhaltens") ist daher nicht nur ein wenig hilfreiches Erklärungsmodell für Adipositas, es ist darüber hinaus schlichtweg falsch!

Gleichzeitig ist die Datenlage hinsichtlich der miserablen Prognose einer konservativen Gewichtsreduktion bestechend. So nehmen je nach Studie zwischen $80 \%$ und $99 \%$ aller Patienten, die auf konservative Weise Gewicht abnehmen, dieses Gewicht im Langzeitverlauf wieder zu (35-42). Ge- 
rade bei Frauen führen Gewichtsreduktionsversuche, die im Jugendalter begonnen wurden, zu einer oft Jahrzehnte lang andauernden Diätspirale mit stetigem Gewichtsanstieg (43). Daher ist die routinemäßig ausgesprochene ärztliche Empfehlung an die übergewichtige Patientin, Gewicht abzunehmen, hoch problematisch!

Beim Lipödem ist der Rat zur Gewichtsreduktion besonders tückisch. Studien der Universität Hohenheim haben ergaben, dass Frauen (im Gegensatz zum Männern) nach einer Gewichtsabnahme überproportional im Bereich der unteren Körperhälfte ihr Gewicht wieder zunehmen (44) - und wie oben dargelegt, nehmen fast alle Diätteilteilnehmer ihr abgenommenes Gewicht wieder zu. Mit anderen Worten: jede ärztliche Empfehlung an die Lipödem-Patientin, Gewicht abzunehmen, erhöht das Risiko einer weiteren Fettgewebszunahme im Bereich der Beine - und damit auch das Risiko einer Zunahme der Beschwerden.

Der „Boom“ des Lipödems liegt u. E. auch im oben skizzierten ärztlichen Umgang mit der von Medizinern besonders ungeliebten Krankheit Adipositas. So hat sich auf Patientenseite das Lipödem in den vergangenen Jahren zu einer "Ausflucht“, zu einer Art „Alibi“, für Gewichtszunahme und für Übergewicht entwickelt. Wenn Adipositas als schuldhaftes Verhalten verurteilt wird, dann bietet - so die unter Patienten populäre Sicht - die Erkrankung Lipödem die Erklärung für die stete $\mathrm{Ge}$ wichtszunahme. „Ich bin nicht dick, ich leide an einem Lipödem“ ist das Credo zahlreicher Lipödem-Selbsthilfegruppen, welches dann auch in den Medien Widerhall findet (45-47).

In der Fachklinik für Lymphologie sind wir täglich mit Lipödempatientinnen konfrontiert, die aufgrund dieser (auf unterschiedlichen Kanälen publizierten) Falschinformation davon überzeugt sind, dass das Lipödem für das seit Jahren progrediente Übergewicht verantwortlich ist. Es ist mühsam und zeitaufwendig, Patientinnen davon zu überzeugen, dass sie falsch informiert wurden. Es ist mühsam und zeitaufwendig, Patientinnen davon zu überzeugen, dass es andere Faktoren sind, die zur Gewichtszunahme führen - sicher aber nicht das Lipödem. Es ist mühsam und zeitaufwendig, Patientinnen davon zu überzeugen, dass die häufig begehrten Therapieoptionen - Manuelle Lymphdrainage oder Liposuktion - mitnichten zu einer substanziellen, nachhaltigen und auch nur annähernd zufriedenstellenden Gewichtsabnahme führen werden.

\section{Fazit}

Es gibt keine Evidenz, dass das Lipödem zu Gewichtszunahme führt. Unsere täglich zahlreich erlebte klinische Erfahrung weist in die gegenteilige Richtung. Gewichtszunahme scheint ein entscheidender Trigger zu sein, um - bei entsprechend genetischer Disposition für ein Lipödem - dieses überhaupt erst zu entwickeln. Hierfür spricht auch die Datenlage unseres klinikinternen Adipositasprogramms, in dem aktuell mehr als 400 Patienten umfassend diagnostiziert und dokumentiert sind (48).

Normal- oder leicht übergewichtige Patientinnen mit starker disproportionaler Fettgewebsvermehrung und Weichteilbeschwerden kommen vor, diese bilden aber angesichts der überwältigenden Mehrheit adipöser bzw. morbid adipöser Lipödempatientinnen eine sehr kleine Minderheit.

\section{Mythos 5: Gewicht abneh- men hat keinen Effekt auf das Lipödem!}

Dieses Statement stellt ein weiteres Dogma innerhalb der Lipödem-Mythologie dar. Diesem Dogma geht nahezu regelhaft die Klage voraus, dass das Lipödem von medizinisch Tätigen meist mit Adipositas verwechselt wird. So schreibt Schmeller: „Oft wird das Lipödem mit der Adipositas verwechselt oder als Teilsymptom der Adipositas angesehen." (49); oder in einer anderen Publikation: „selbst von Ärzten und medizinischem Fachpersonal wird die Erkrankung häufig übersehen bzw. mit einer Adipositas oder einem Lymphödem verwechselt" (50). Ähnlich besorgt schreiben auch Cornely und Gensior auf ihrer Website: „Lipödeme werden selten schnell und richtig diagnostiziert. Oftmals müssen sich die Patientinnen anhören: Sie sind zu dick“" (51).
Karen Herbst, Endokrinologin und Protagonistin der amerikanischen Lipödemszene titelt ihren Artikel, der im Wesentlichen das Lipödem zum Thema hat: „Rare adipose disorders (RADs) masquerading as obesity". Darin schreibt sie:

"One of the most common misconceptions about patients with lipedema is that they suffer, instead, from lifestyle- or dietinduced obesity"; und an anderer Stelle: Lipedema "may be misdiagnosed as obesity“ (52).

Diese Sicht auf das Lipödem wird gerne auch von den Medien sowie von Patientenseite übernommen. So meint das Magazin Stern: „Frauen, die unter einem Lipödem leiden, werden meist fälschlicherweise für schlicht übergewichtig gehalten und eine über das Lipödem schreibende Schauspielerin (deren Buch zu einem Bestseller der deutschen Lipödemszene aufgestiegen ist) glaubt, dass viele Lipödempatientinnen in Arztpraxen als „gar adipös gesehen werden" (53-54). Unnötig zu erwähnen, dass Lipödem-Selbsthilfe-Gruppen und Foren im Internet dieser populären Auffassung folgen (55).

Es kann nicht deutlich genug betont werden: Die große Mehrheit der Lipödempatientinnen ist tatsächlich AUCH adipös!

Um die oben bereits genannten Zahlen noch einmal zu wiederholen: Tatsächlich ist die normalgewichtige Lipödempatientin eine Rarität! In unserem ambulant gesehenen Patientengut 2015 (über 2300 Patientinnen) waren nur $3 \%$ der Lipödempatientinnen normalgewichtig; ähnliche Zahlen liegen aus England und aus den Niederlanden vor. Mit anderen Worten: In weit überwiegender Zahl leiden diese Patientinnen an zwei Problemen, an Adipositas UND an einem Lipödem. Die Fokussierung auf das Lipödem bei einer Patientin mit einem BMI von 35 oder 45 oder $55 \mathrm{~kg} / \mathrm{m}^{2}$ erscheint uns grotesk und wenig zielführend, das Auseinanderdividieren beider Probleme als nicht hilfreich. Viele unserer Lipödempatientinnen leiden nach unserer Erfahrung häufig stärker unter der progredienten Gewichtszunahme (und der damit im Zusammenhang stehenden „aktuell nicht angesagten Körperkonfiguration") als unter den tatsächlichen Schmerzen in den Beinen. 
Konsequenz dieser von vielen Lipödemexperten - und in Folge dessen auch von Lipödempatientinnen - propagierten Sicht, ist die Annahme, dass Gewichtsabnahme auch keinen wesentlichen Einfluss auf das Lipödem (bzw. auf die im Rahmen des Lipödems auftretenden Beschwerden) habe. Es würde sich lediglich das „Adipositasfett" nicht aber „Lipödemfett" verringern. So schreibt die oben bereits zitierte Endokrinologin Herbst: „Although lifestyle changes and bariatric surgery work effectively for the obesity component"... „these treatments do not routinely reduce the abnormal subcutaneous adipose tissue " of Lipedema (56).

Auch Schmeller stellt fest: Gewichtsabnahme helfen ,allerdings nur bei Adipositas und bewirken lediglich eine Umfangsreduktion am Stamm. Da die Lipödemspezifischen Fettvermehrungen aber nicht ,abhungerungsfähig' sind, verstärkt sich die Diskrepanz zwischen Rumpf und Extremitäten noch mehr" (57). Unter der Überschrift: „Die Fakten sind also" behauptet Cornely: „Eine Verbesserung der Beschwerden durch Diät und Sport ist nicht möglich“ (58). Dass es sich bei den Fettpolstern an den Beinen nicht wie bei der Fettleibigkeit um gespeichertes Reservefett handelt, davon ist Stutz, ein (wie auch Schmeller und Cornely) die Fettabsaugung durchführender Arzt, überzeugt. In seinem Patientenflyer hält er fest: „Dieses Fett hat eine gänzlich andere Struktur und kann daher nicht durch Diät und Sport abgenommen werden" (59).

Es ist auffällig - und diese Anmerkung sei erlaubt - wie stark sich in Deutschland vor allem Liposuktionen durchführende Ärzte für diese Betrachtungsweise engagieren. So finden sich auch auf den Websites von weiteren fettabsaugenden Kollegen der Hinweis, dass Sport und Gewichtsabnahme das Lipödem nicht positiv beeinflussen (z. B. 60-62).

Diese Sicht der Dinge wird auch wird auch von Patientinnen und LipödemSelbsthilfegruppen dankbar übernommen. So betrachtet das von Lipödempatientinnen häufig im Internet besuchte LipödemPortal die erfolglose Gewichtsreduktion als „Leitsymptom“ des Lipödems: „Die Menge des Fettgewebes im Lipödem kann weder durch Diät noch Sport reduziert werden
(Diät-Resistenz). Selbst extreme Abmagerungskuren, die Oberkörper und Gesicht ausgemergelt und knochig wirken lassen, führen zu keiner Abnahme des Lipödems (Leitsymptom)" (63, Hervorhebung im Original).

Einmal abgesehen von der Tatsache, dass weder die Autoren noch (auf Nachfrage der Autoren) Oberärzte und Chefarzt der Fachklinik für Lymphologie in den vergangenen Jahrzehnten ,ausgemergelte und knochig wirkende" Lipödempatientinnen zu Gesicht bekamen, und im Netz hierzu lediglich ein einziges und immer wieder gezeigtes Foto kursiert (96), bleibt doch die Frage nach der wissenschaftlichen Evidenz dieser bei Experten und Patientinnen so populären Sichtweise.

Welches pathophysiologische Konstrukt liegt der Annahme zugrunde, dass die adipöse, an Lipödem erkrankte Patientin bei einer Gewichtsabnahme nicht auch im Bereich der Extremitäten substanziell Fettgewebe verlieren sollte?

Es bleibt festzuhalten, dass auch die o. g. Autoren in ihren Veröffentlichungen kein Erklärungsmodell liefern, welches auch nur im Ansatz die von ihnen publizierten Statements begründet. Insofern bleiben es Mythen, Mythen, die seit Jahren tradiert und von Lipödempatientinnen übernommen werden. Über die Gründe, warum gerade die Liposuktion ausübenden Ärzte an der Verbreitung dieser Mythen erheblichen Anteil haben, darf spekuliert werden.

Diesen Mythen gegenüber stehen die historischen Publikationen der Erstbeschreiber des Lipödems Allen and Hines aus den Jahren 1940 und 1951. Dort wird festgestellt: „In cases of generalized obesity, sharp reduction of weight may help“ (64-65).

Diese Beschreibung der Erstautoren deckt sich auch mit der seit vielen Jahren bestehenden klinischen Erfahrung, die wir und unsere Kollegen in der Fachklinik für Lymphologie mit Lipödempatientinnen machen.

Immer wieder sehen wir Lipödempatientinnen, denen - zumindest kurzfristig - eine Gewichtsabnahme auf konservative Weise gelungen ist und die dadurch auch eine - zumindest passagere - Fettgewebsreduzierung im Bereich der Extremitäten erfahren (bei jedem Patient in der Földikli- nik werden - neben der Messung Größe, Gewicht, BMI, Bauchumfang und waist-toheight-Index - detaillierte Umfangsvermessungen an Armen und/oder Beinen vorgenommen).

Nachhaltiger und substanzieller sind Gewichtsabnahmen, die Patientinnen im Rahmen unseres multimodalen Adipositasprogramms erfahren haben. In diesem Programm werden Patienten mit Lymphödem aber auch mit Lipödem ab einem BMI von $40 \mathrm{~kg} / \mathrm{m}^{2}$ - Eignung und Einverständnis vorausgesetzt - für einen adipositaschirurgischen Eingriff vorbereitet. Das Adipositasprogramm der Földiklinik wurde bereits in einer früheren Ausgabe dieser Zeitschrift skizziert (66). Die hierdurch erreichte Gewichtsreduktion schlägt sich regelhaft auch in einer meist proportionalen Umfangsverminderung der Extremitäten wider.

Mit anderen Worten: Durch die im Rahmen einer bariatrischen Operation (Magenbypass oder Schlauchmagen) erreichte Gewichtsabnahme nehmen Patientinnen mit Lipödem substanziell und meist nachhaltig auch im Bereich ihrer Arme und Beine ab. Eine gewisse Disproportionalität der Beine verbleibt meist nach erfolgter Gewichtsabnahme. Allerdings stellt die alleinige beschwerdefreie Disproportionalität der Beine in der Regel keinen krankhaften Zustand dar. Ziel dieser medizinischen Therapie ist nicht ein Bein, welches dem aktuellen Schönheitsideal nahekommt - dies wäre ggf. Aufgabe einer kosmetischen Operation, die dann selbstverständlich auch nicht zu Lasten der Allgemeinheit durchzuführen wäre! Ziel dieser medizinischen Therapie ist in erster Linie Beschwerdebesserung bzw. Beschwerdefreiheit. Die weit überwiegende Mehrheit unserer Patientinnen erlebt infolge der oft erheblichen Fettgewebsreduktion an den Beinen eine deutliche Beschwerdebesserung, viele sind sogar schmerzfrei. Wir sprechen dann von einem „Lipödem in Remission". Bleibt der Gewichtsverlauf auch in den Folgejahren weitgehend stabil, ist nicht zu erwarten, dass sich erneut eine Beschwerdesymptomatik entwickelt. Aus einem Lipödem wird somit eine - meist nur noch milde - Lipohypertrophie, die keinerlei Krankheitswert hat. Aktuell werden diese seit vielen Jahren bestehenden positiven 
Erfahrungen der Gewichtsabnahme auf das Lipödem im Rahmen einer Studie mit der Universität Freiburg untersucht.

Adipösen Patienten mit einem BMI unter $40 \mathrm{~kg} / \mathrm{m}^{2}$ oder Patienten, bei denen (z.B. aufgrund internistischer oder psychologischer Kontraindikationen) ein adipositaschirurgischer Eingriff nicht möglich ist, raten wir zur langfristigen Gewichtsstabilisierung. Gleichzeitig raten wir explizit von Diäten oder konservativen Gewichtsreduktionsprogrammen ab. Wir begründen dieses Vorgehen mit der desaströsen Langzeitprognose von konservativen Gewichtsreduktionsversuchen, die durch die oben bereits erwähnte konsistente Datenlage belegt ist. Diese konsistente Datenlage deckt sich auch mit unserer klinischen Erfahrung mit Patienten, die wir seit vielen Jahren lymphologisch betreuen und die im Rahmen ihrer oft Jahrzehnte andauernden „Diätkarriere" stetig an Gewicht zugenommen haben. Statt "diet and exercise" lautet das Credo unserer Klinik daher „stabilize and exercise“. Darüber hinaus ist - neben der für alle Lipödempatientinnen essenziellen Kompressionstherapie - für diese Patientengruppe die Förderung der Selbstakzep$\tan z$ - insbesondere die Akzeptanz des eigenen Körpers - von herausragender Bedeutung. Erst wenn diese gelingt, ist ein Ausstieg aus dem Teufelskreis von Diäten, Jo-Jo-Effekt und Verschlechterung des Lipödems möglich.

Bariatrisch operierte Patientinnen mit Lipödem erleben über die Verbesserung ihrer Lipödembeschwerden hinaus aber auch weitere positive Auswirkungen: So erfahren diese Patientinnen eine deutliche Steigerung ihrer Mobilität und damit auch ihrer Lebensqualität (67-68).

Hinzu kommt der kardiovaskuläre Benefit des adipositaschirurgischen Eingriffes: Diabetes mellitus, Bluthochdruck und Schlafapnoesyndrom bilden sich partiell, oft auch komplett, zurück (69-73).

Schließlich zeigen mehrere große Studien die Effektivität bariatrischer Operationen hinsichtlich der Reduktion der Langzeit-Mortalität auf: Patienten nach Schlauchmagen oder Magenbypass-Operationen leben länger und gesünder als vergleichbar adipöse Patienten ohne adipositaschirurgischen Eingriff (74-77).
Die von Ernährungsmedizinern häufig gehörte Behauptung, dass der metabolische Erfolg von konservativen Ernährungsprogrammen mit denen von operativen nach Schlauchmagen oder Magenbypass vergleichbar sind, sind u.E. eher einem Wunschdenken geschuldet als wissenschaftlicher Evidenz. So schreibt G. Faerber in dieser Zeitschrift: „Diese Ergebnisse sind also mit denen nach bariatrischen Operationen vergleichbar" (78). Als Grundlage dieses Statements dienen der Autorin zwei Untersuchungen, mit extrem niedriger Fallzahl (11 bzw. 29 Probanden!) und ebenso extrem kurzer Studiendauer (maximal 8 Wochen!). Die Probanden mussten sich während dieser acht Wochen einer 600 kcal-Diät (Optifast von Fa. Nestle) unterziehen $(79,80)$. Danach wurden diverse metabolische Parameter untersucht. Offen bleibt die Frage, wie lange die Probanden diese 600 kcal-Diät nach Studienende fortgeführt haben und wie sich die metabolischen Parameter dann weiterentwickelt haben.

Diese dünne Studienlage mit Ergebnissen zur bariatrischen Operation zu vergleichen, Studien, die, wie oben genannt (69-71, 73-77), über mehrere Jahre Tausende Patienten untersucht haben, ist erstaunlich und letztlich nur verständlich, wenn man die kritische Haltung von Faerber zu operativen Therapieoptionen teilt. Das Scheitern von konservativen Gewichtsreduktionsprogrammen im Langzeitverlauf ist durch zahlreiche - hochwertige - Studien dokumentiert (81-91) und wurde bereits von Bertsch (2) in dieser Zeitschrift beschrieben. Weiter warnt die Autorin eindringlich vor „Fehl- oder Mangelernährung, Osteoporose, Ösophagitis“ und mehr. Eine umfassendere Ausbildung von Ernährungsmedizinern und Diätassistenten in der Kunst der postbariatrischen Nachsorge wäre hier u. E. die bessere Antwort als das pauschale Abraten vor einer Therapieoption, die sehr vielen Patienten die Chance auf ein gesünderes, längeres und leichteres Leben gibt.

Darüber hinaus warnt Faerber in ihrem Beitrag vor einer erhöhten Suizidrate in Folge von bariatrischen Operationen. Im gleichen Artikel dieser Zeitschrift (78) bezieht sie sich dabei auf die Studie von Tindle (92) in den USA. Auch in einem Review von Peterhänsel (93) wird eine gegenüber der Allgemeinbevölkerung (!) erhöhte Suizidrate beschrieben.

Bei kritischer Reflexion wird schnell klar, dass hier ein völlig ungeeigneter Vergleich herangezogen und nicht Vergleichbares aufeinander bezogen wird: eine Gruppe bariatrisch operierter Patienten wird einer - nach Alter und Geschlecht parallelisierten - Gruppe aus der Normalbevölkerung gegenübergestellt (92). Der weitaus bedeutsamere BMI wird jedoch völlig außer Acht gelassen. Dabei ist bekannt, dass gerade Personen mit einem höheren BMI auch eine höhere Komorbidität psychischer Erkrankungen aufweisen (94).

Werden jedoch Adipöse, die sich auf einen bariatrischen Eingriff vorbereiten, verglichen mit einer Gruppe Adipöser aus der Bevölkerung, so unterscheiden sich beide Gruppen nicht hinsichtlich ihres selbstschädigenden Verhaltens oder der Häufigkeit von Suizidversuchen (95). Die vermeintlich erhöhte Suizidalität ist also nicht - wie oft fälschlicherweise angenommen Folge der bariatrischen Operation, sondern vielmehr Folge der erhöhten psychischen Vulnerabilität der Patientengruppe mit ausgeprägter Adipositas.

So schreiben auch Tindle et al (92) schließlich zur Erklärung der Suizide: „Suicides are not necessarily attributed to the bariatric surgery, but may be related to myriad factors."

Faerbers Argumentation läuft daher ins Leere. Davon unabhängig kann die Bedeutung einer umfassenden Vorbereitung und Nachsorge von Patienten, die sich einem adipositaschirurgischen Eingriff unterziehen, nicht genug betont werden - auch um jene Personen $\mathrm{zu}$ identifizieren, die psychotherapeutischer Unterstützung bedürfen und diese zeitnah qualifiziert zu behandeln.

Die Therapie von morbid adipösen Menschen ist nur dann langfristig erfolgreich, wenn spezialisierte Berufsgruppen gemeinsam an einem Strang ziehen: Ernährungsmediziner wie Psychotherapeuten, Internisten und Allgemeinmediziner wie Chirurgen.

- Abbildungen 1 und 2 zeigt eine Lipödempatientin (Gewicht $122 \mathrm{~kg}$, Größe 168 $\mathrm{cm}$, BMI $43 \mathrm{~kg} / \mathrm{m} 2$ ) vor Schlauchmagen- 


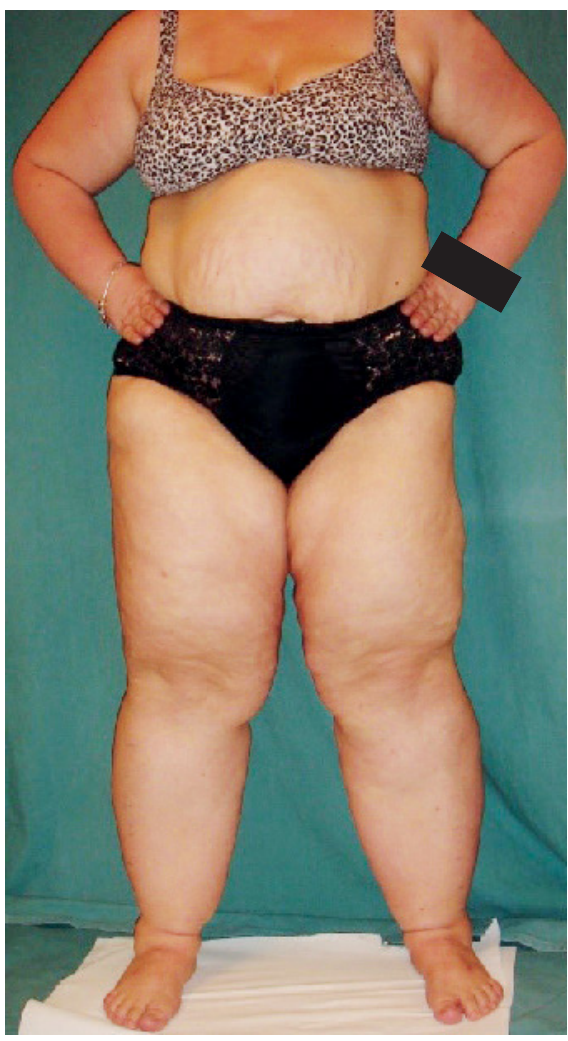

Abb. 1 Lipödempatientin (Gewicht 122 kg, GröBe $168 \mathrm{~cm}$, BMI $43 \mathrm{~kg} / \mathrm{m}^{2}$ ) vor Schlauchmagenoperation. Das Beinvolumen beträgt hier je Bein 19 Liter)

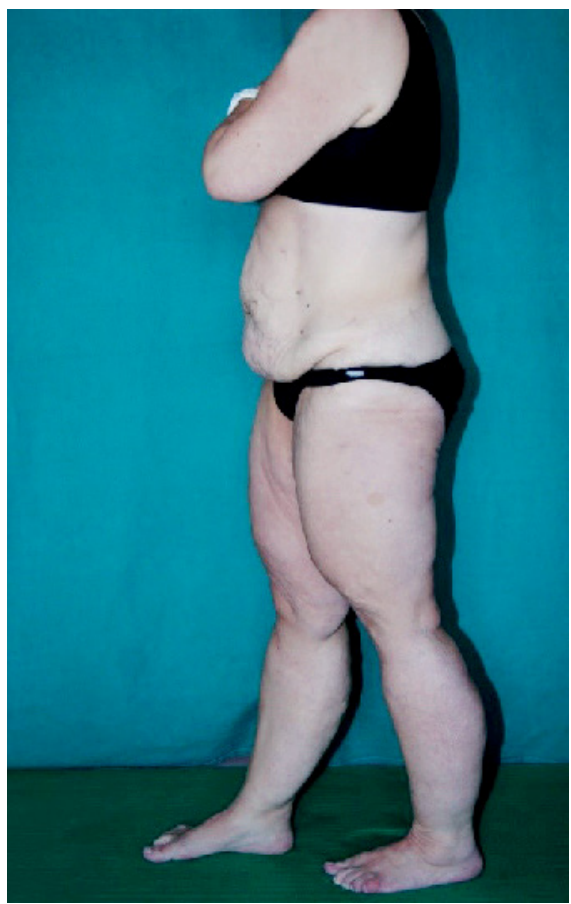

Abb. 3 Patientin aus Abb. 1 und 2, 11 Monate nach erfolgter bariatrischer OP. Das Gewicht betrug jetzt $74 \mathrm{~kg}$, der BMl lag bei $26 \mathrm{~kg} / \mathrm{m}^{2}$.

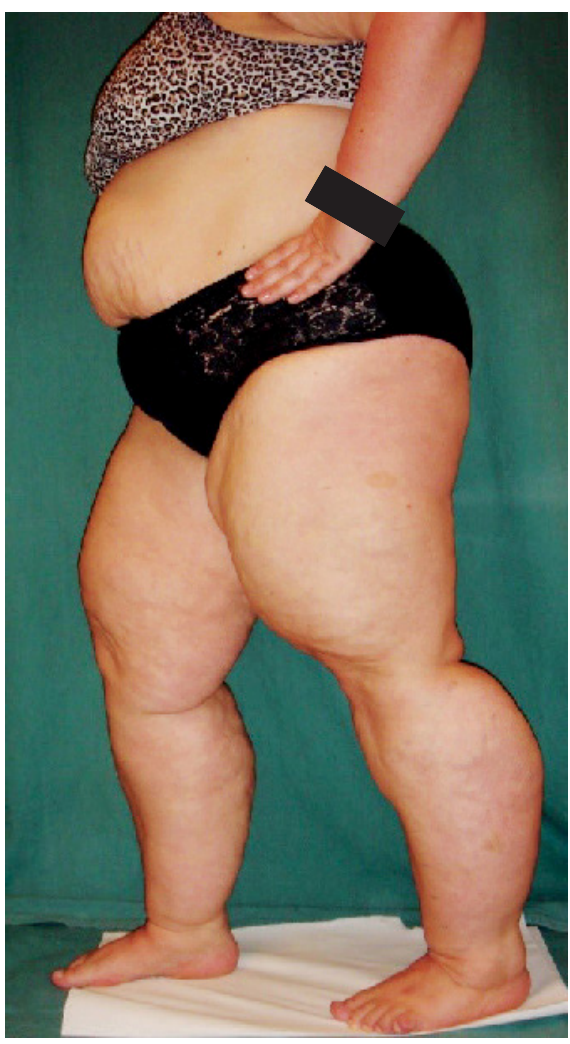

Abb. 2 Lipödempatientin (Gewicht 122 kg, GröBe $168 \mathrm{~cm}, \mathrm{BMI} 43 \mathrm{~kg} / \mathrm{m}^{2}$ ) vor Schlauchmagenoperation. Das Beinvolumen beträgt hier je Bein 19 Liter)

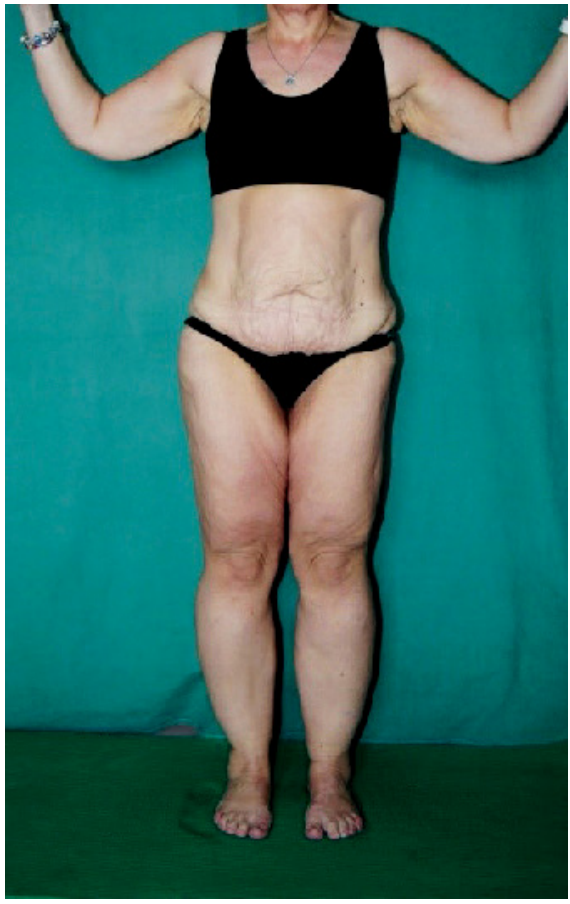

Abb. 4 Patientin aus Abb. 1 und 2, 11 Monate nach erfolgter bariatrischer OP. Das Gewicht betrug jetzt $74 \mathrm{~kg}$, der BMl lag bei $26 \mathrm{~kg} / \mathrm{m}^{2}$. operation. Das Beinvolumen beträgt hier je Bein 19 Liter)

In $>$ Abbildungen 3 und 4 sehen wir die gleiche Patientin 11 Monate nach erfolgter bariatrischer OP. Das Gewicht betrug jetzt $74 \mathrm{~kg}$, der BMI lag bei $26 \mathrm{~kg} / \mathrm{m}^{2}$. Das Beinvolumen je Bein betrug jetzt noch 9 Liter. Die Patientin war komplett beschwerdefrei, sodass wir hier von einem „Lipödem in Remission" sprechen. Eine plastisch-chirurgische Hautstraffung der Oberschenkel war bei dieser Patientin nicht zwingend notwendig.

- Abbildung 5 zeigt eine Patientin mit Lipödem und inzwischen auch distal betontem -Adipositas-assoziierten - Beinlymphödem vor einer Magenbyass-OP, - Abbildung 6 die gleiche Patientin ein Jahr später. Es besteht allenfalls noch eine milde und selten auftretende Lipödembeschwerdesymptomatik; auch das Adipositas-assoziierte Beinlymphödem hat eine massive Besserung erfahren. Die überschüssige Haut mit Unterhautfettgewebe wird durch Abbildung 7 deutlich. Nächster Schritt ist hier jetzt die Dermatolipektomie durch den Plastischen Chirurgen.

Die Lipödempatientin in $>$ Abbildungen 8 und 9 nahm im Rahmen einer Schlauchmagen-Operation binnen von 14 Monaten $65 \mathrm{~kg}$ Gewicht ab. Nach Gewichtsstabilisierung von knapp einem Jahr erfolgte eine Straffung der den Genitalbereich überlagernden Bauchschürze sowie eine Oberschenkelstraffung durch den plastischen Chirurgen. Lipödembeschwerden bestanden keine mehr, sodass wir auch hier von einem Lipödem in Remission sprechen. Im Fall dieser Patientin liegt auch sicher keine Lipohypertrophie mehr vor.

\section{Fazit}

Es gibt weder eine wissenschaftliche noch eine empirische Evidenz für das populäre Statement, dass Gewichtsabnahme nicht zu einer Besserung des Lipödems führt. Unsere seit nunmehr 10 Jahren bestehende klinische Erfahrung weist auf das Gegenteil hin. Nachhaltige Gewichtsabnahme führt $\mathrm{zu}$ einer deutlichen Besserung der Beschwerden; häufig sind die Patienten auch 


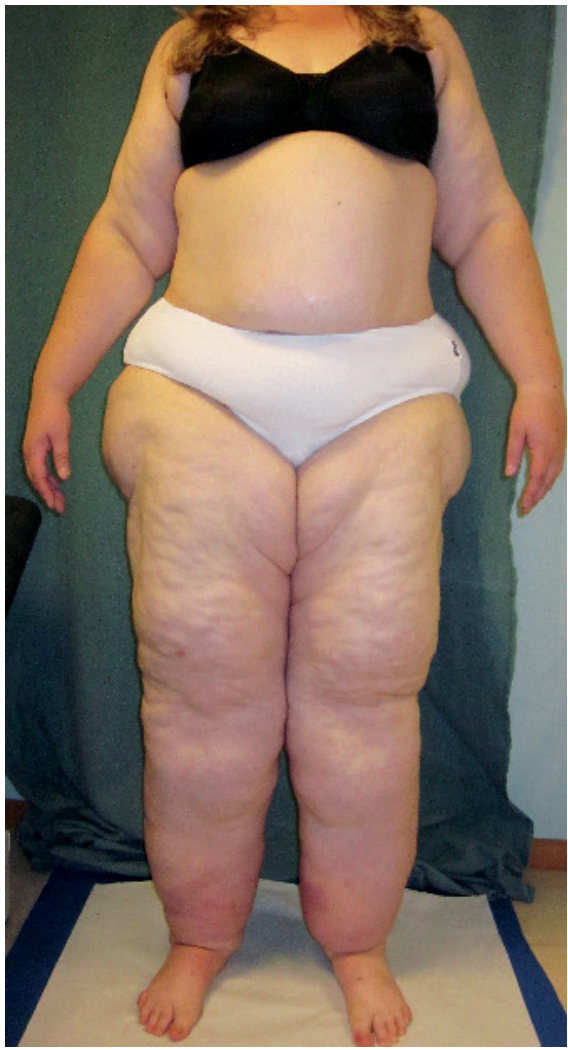

Abb. 5 Patientin mit Lipödem und inzwischen auch distal betontem Beinlymphödem

beschwerdefrei. Wir sprechen dann von einem Lipödem in Remission.

\section{Ausblick}

In einem vierten Beitrag werden wir uns mit dem Stellenwert der Liposuktion beim Lipödem beschäftigen, um dann in unserer letzten Darstellung ein therapeutisches Konzept vorzustellen, dass nicht nur wissenschaftlich fundiert ist, sondern auch zu einer nachhaltigeren und umfassenderen Beschwerdebesserung unserer Lipödempatientinnen beitragen soll.

\section{Interessenkonflikt}

Nach Angaben der Autoren bestehen keine Interessenkonflikte.

\section{Ethische Richtlinien}

Für das Manuskript wurden keine Studien an Menschen oder Tieren durchgeführt.

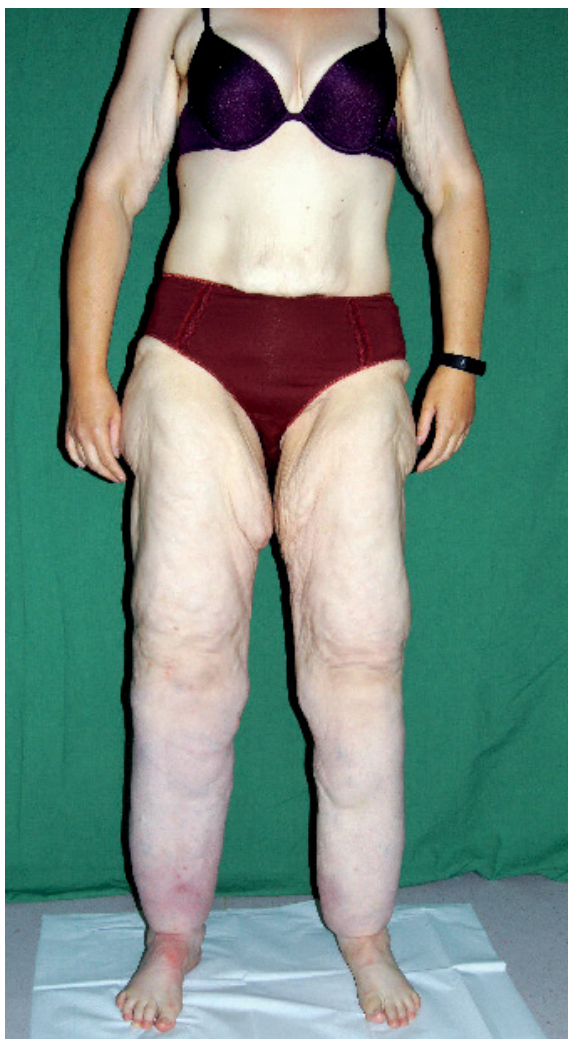

Abb. 6 Patientin aus Abb. 5, ein Jahr später, nach Magenbypass-OP

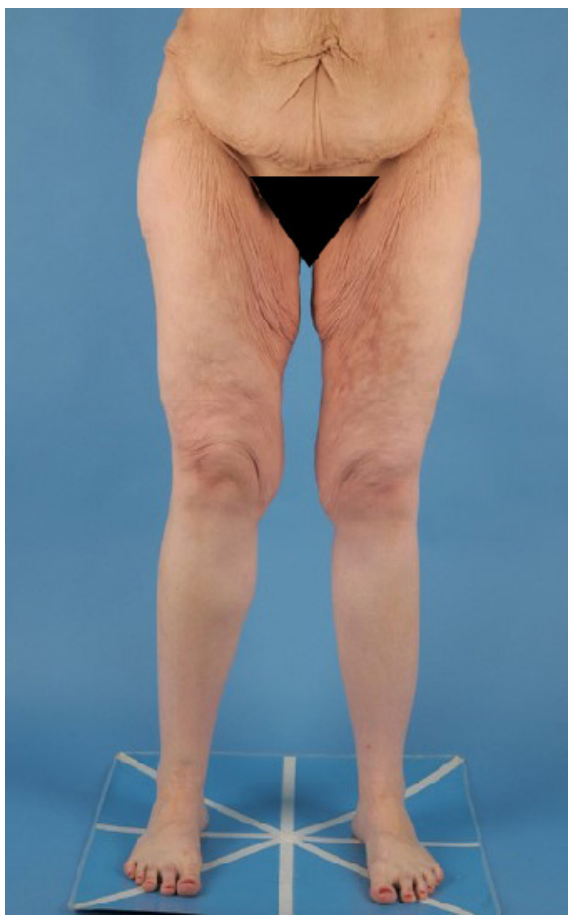

Abb. 8 Lipödempatientin, die im Rahmen einer Schlauchmagen-Operation binnen 14 Monaten 65 kg Gewicht abnahm (Abb. 8 und Abb. 9: mit freundl. Genehmigung Prof. Dr. N. Torio, Freiburg)

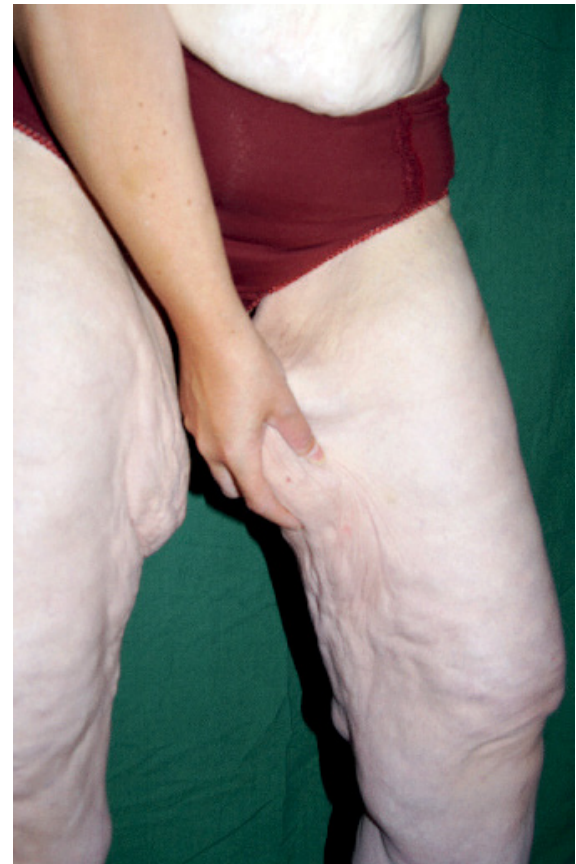

Abb. 7 Patientin aus Abb. 5, ein Jahr später. Überschüssige Haut mit Unterhautfettgewebe

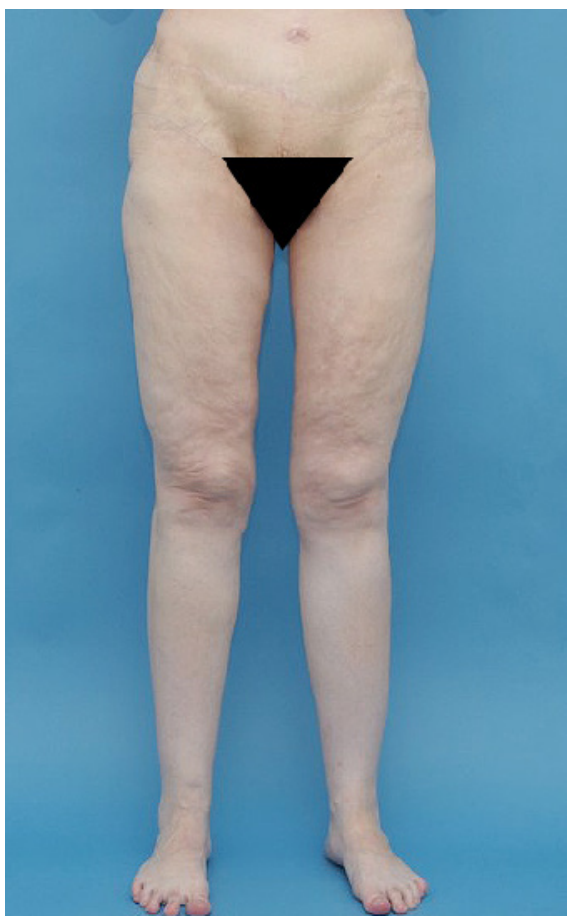

Abb. 9 Nach Gewichtsstabilisierung von knapp 1 Jahr erfolgte eine Straffung der den Genitalbereich überlagernden Bauchschürze und eine Oberschenkelstraffung durch plastischen Chirurgen 


\section{Literatur}

1. Bertsch T, Erbacher G. Lipödem - Mythen und Fakten Teil 1. Phlebologie 2018; 47: 84-92.

2. Bertsch T. Adipositas-assoziierte Lymphödeme unterschätzt und unterbehandelt. Phlebologie 2018; 47: 75-83.

3. Bertsch T, Erbacher G. Lipödem - Mythen und Fakten Teil 1. Phlebologie 2018; 47: 84-92.

4. Bertsch T, Erbacher G. Lipödem - Mythen und Fakten Teil 2. Phlebologie 2018; 47: 120-126.

5. Bertsch T, Martin KP. Adipositasprävalenz unter Lipödempatientinnen in einer kassenärztlichen lymphologischen Ambulanz im Jahr 2015 (unveröffentlichte Daten)

6. Bosman J. Lipoedema: Poor knowledge, neglect or disinterest? Journal of Lymphoedema, 2011, Vol 6, No 2, 109-111.

7. Child AH. Lipedema: an inherited condition. Am J Med Genet A. 2010;152A(4):970-976.

8. STERN TV. Was Sie über Lipödeme wissen sollten. Abrufbar unter: https://www.stern.de/tv/faqkrankhafte-fettverteilung--was-sie-ueber-lipoedeme-wissen-sollten-7339120.html

9. Sputnik.de. Mysteriöse Krankheit, die dick macht: Was ist Lipödem? Abrufbar unter: https://www. sputnik.de/sendungen/tagesupdate/lipoe dem-100.html

10. Evidero.de. Dick durch Krankheiten und Medikamente: Dick oder krank? Diese Krankheiten beeinflussen Gewicht und Aussehen. Abrufbar unter: https://www.evidero.de/krankheiten-beein flussen-das-gewicht

11. NDR.de. Markt vom 12.03.2018. Lipödem: Kasse zahlt Behandlung nicht. Abrufbar unter: https://www.ndr.de/fernsehen/sendungen/markt/ Seltene-Krankheit-Kasse-zahlt-Behandlungnicht,markt12014.html

12. YouTube. Lipödem im Bauch - gibt's das überhaupt? JA! Abrufbar unter: https://www.youtube. com/watch?v=tjbdntLfo2Q

13. Herbst KL. Obesity and Lipedema - What's the link? Abrufbar unter http://www.obesityaction. org/wp-content/uploads/Lipedema_and_Obesity_online.pdf

14. Obesityhelp.com. Could you have Lipedema? Abrufbar unter: http://www.obesityhelp.com/articles/ could-you-have-lipedema

15. Stunkard AJ et al. An Adoption Study of Human Obesity. N Engl J Med 1986; 314: 193-198

16. Stunkard AJ et al. The Body-Mass Index of Twins who have been reared apart. N Engl J Med 1990; 322: 1483-1487.

17. Plagemann A (Ed.). Perinatal Programming The State of the Art. Berlin/Boston: Walter de Gruyter 2012; 11-22.

18. Herrera B et al. Genetics and epigenetics of obesity. Maturitas 2011; 69 (1): 41-49.

19. Hewagalamulage SD et al. Stress, Cortisol and obesity: a role for cortisol responsiveness in identifying individuals prone to obesity. Domest Anim Endocrinol 2016; 56 Suppl: 112-120.

20. Volkow ND et al. Obesity and addiction: neurobiological overlaps. Obesity reviews 2013 (1): 2-18

21. Nemiary D et al. The Relationship Between Obesity and Depression Among Adolescents. Psychiatr Ann. 2013; 42 (8): 305-308.
22. Seese B. Pathophysiologie der Adipositas. Abrufbar unter: https://www.cme-kurs.de/cdn2/pdf/ Handout_Adipositas.pdf

23. Taubes G. The Case against Sugar. New York: Alfred A Knopf 2016.

24. Malik, V.S. \& Hu, F.B. Sweeteners and Risk of Obesity and Type 2 Diabetes: The Role of SugarSweetened Beverages. Curr Diab Rep (2012) 12: 195.

25. Wang JW. Consumption of added sugars and development of metabolic syndrome components among a sample of youth at risk of obesity. Applied Physiology, Nutrition, and Metabolism, 2014, 39(4) 512

26. Pietiläinen $\mathrm{KH}$ et al. Does Dieting Make You Fat? International J of Obesity 36 (3) 2012. 456-464

27. Robert Koch-Institut 2016. Studie DEGS1, Erhebung 2008-2011.

28. Sifferlin A. $40 \%$ of Americans Are Obese - And the Trend Isn't Slowing. In TIME HEALTH, October 2017. Abrufbar unter: http://time. com/4980225/obesity-rates-adults-children/

29. WHO. Controlling the global obesity epidemic, 2017. Abrufbar unter: http://www.who.int/nutri tion/topics/obesity/en/

30. Hilbert A et al. Stigmatisierung bei Adipositas. In Adipositas 2013; 3: 150-153.

31. Jung FU et al. Stigma and Knowledge as Determinants of Recommendation and Referral Behavior of General Practitioners and Internists. Obes Surg 2016; 26: 2393-2401.

32. Kimberly AG et al. Physicians build less rapport with obese patients 2013; 21 (10): 2146-2152.

33. Brownell KD et al. Weight Bias: Nature, consequences and remedies. New York: Guilford Press 2005.

34. Hilbert A, Geisert M. Stigmatisierung von Adipositas: Implikation für die Kommunikation mit adipösen Patienten. In: Lewandowski K, Bein T (Hrsg). Adipositas in Anästhesie, Intensiv- und Notfallmedizin. 2012. Medizinisch-Wissenschaftliche Verlagsgesellschaft 71-77.

35. Stunkard A. The Results of Treatment for Obesity. A Review of the Literature and Report of a Series. AMA Arch Intern Med 1959; 103(1): 79-85.

36. Bennett W, Gurin J. The Dieter's Dilemma: Why Diets Are Obsolete-the New Setpoint Theory of Weight Control. New York: Basic Books 1982.

37. Cogan J, Rothblum E. Outcomes of weight-loss Programms. Genetic, Social and General Psychology Monographs 1993; 118 (4).

38. Perri MG. et al. Sucess and failure in the treatment of obesity: Where do we go from here? Medicine, Exercise, Nutrition and Health 1995; 4: 255-272.

39. Hensrud DD, Weinsier RL. A prospective study of weight maintenance in obese subjects reduced to normal body weight without weight-loss training. American clinical Nutrtion 1994; 60(5): 688-694

40. Mann T, Tomiyama AJ et al. Medicare's search for effective obesity treatments: diets are not the answer. Am Psychol 2007; 62(3): 220-233.

41. Nordmann A et al. Effects of low carb vs low fat diets on weight loss and cardiovascular risk factors: a meta analysis of randomized controlled trials. Arch Internal Med 2006; 166 (8).

42. Fildes A, Charlton J. Probability of an obese person attaining normal body weight. American Journal of Public Health 2015.
43. Pietiläinen KH et al. Does Dieting Make You Fat? AAO.

44. Bosy-Westhpal A. Effect of weight loss and regain on adipose tissue distribution, composition of lean mass and resting energy expenditure in young overweight and obese adults. International Journal of Obesity 2013; 1-7.

45. Goddard S. I'm not fat, I've got lipedemda. BBC. com Abrufbar unter: http://www.bbc.co.uk/ bbcthree/item/ 5d58f224-4bf7-498f-97d4-4073754a8f7f

46. Seo C. Movie: The disease they call fat. Abrufbar unter. https://diseasetheycallfat.tv

47. Baldzun A. Diagnose Lipödem: Der tägliche Kampf mit dem krankhaften Fett. Fokus am 24.1.2017. Abrufbar unter: https://www.focus.de/ gesundheit/ratgeber/frauenmedizin/mehr-alsdrei-millionen-betroffene-diagnose-lipoedemder-taegliche-kampf-mit-dem-krankhaftenfett_id_6522464.html

48. Adipositasprogramm der Földiklinik. Unveröffentlichte Patientendaten

49. Schmeller W, Meier-Vollrath I. Lipödem - Aktuelles zu einem weitgehend unbekannten Krankheitsbild. Akt Dermatol 2007; 33(7): 251-260.

50. Schmeller W, Meier-Vollrath I. Moderne Therapie des Lipödems: Kombination von konservativen und operativen Maßnahmen. LymphForsch 2004; 8(1): 22-26.

51. Cornely M, Gensior M. Website abrufbar unter: http://www.cg-lympha.de/de/krankheitsbilder/li poedem

52. Herbst KL. Rare adipose disorders (RADs) masquerading as obesity. Acta Pharmacologica Sinica Volume 2012; 33: 155-172.

53. Stern TV. Was Sie über Lipödeme wissen sollten. Abrufbar unter: https://www.stern.de/tv/faqkrankhafte-fettverteilung--was-sie-ueber-lipoedeme-wissen-sollten-7339120.html

54. Kaniuth M. Dicke Beine trotz Diät: Mein Leben mit Lipödem. 2015. MGV-Verlag München S. 9

55. Lipödemportal.de. Alles über das Lipödem. Abrufbar unter: http://www.lipoedemportal.de/lipoe dem-probleme.htm

56. Herbst KL. Rare adipose disorders (RADs) masquerading as obesity. AAO. S. 155.

57. Schmeller W, Meier-Vollrath I. Lipödem - Aktuelles zu einem weitgehend unbekannten Krankheitsbild. AAO. S. 256.

58. Cornely M. Das Lipödem an Armen und Beinen. Teil 2: Zur konservativen und operativen Therapie des Lipödems, genannt Lipohyperplasia dolorosa. Phlebologie 2011; 40: 146.

59. Website Dr. Stutz. Abrufbar unter: http://stutz-dr. com/wp-content/uploads/2018/01/DownloadFile-1.pdf

60. Website Rosenbergklinik. Abrufbar unter: http://www.rosenbergklinik.de/index.php?id=28

61. Website LipoClinic Dr. Heck. Abrufbar unter: http://www.falk-heck.de/krankheitsbild-lipoe dem-ursachen-problem-untersuchungen-stadien.html

62. Website Lipocura. Abrufbar unter: https://lipocu ra.de/lipoedem/diagnose/

63. Lymphverein.de. Die typischen Kennzeichen des Lipödems. Abrufbar unter: http://www.lymph verein.de/lipoedem.html 
64. Allen E, Hines E. Lipedema of the legs: a syndrome characterized by fat legs and orthostatic edema. Proc Staff Mayo Clin 1940; 15: 184-187.

65. Wold LE, Hines EA, Allen EV. Lipedema of he legs; a syndrome characterized by fat legs and edema. Ann Intern Med 1951; 34(5): 1243-1250.

66. Bertsch T. Adipositas-assoziierte Lymphödeme unterschätzt und unterbehandelt. AAO.

67. Herpertz S, Kielmann R, Wolf AM, et al. Does obesity surgery improve psychosocial functioning? A systematic review. Int J Obes Relat Metab Disord 2003; 27: 1300-1314.

68. Buddeberg-Fischer B, Klaghofer R, Sigrist S, et al. Impact of psychosocial stress and symptoms on indication for bariatric surgery and outcome in morbidly obese patients. Obes Surg 2004; 14: 361-369.

69. Buchwald H, Estok R, Fahrbach K, et al Weight and type 2 diabetes after bariatric surgery: systematic review and meta-analysis. Am J Med 2009; 122: 248-256.

70. Wittgrove AC, Clark GW. Laparoscopic Gastric Bypass, Roux en-Y - 500 Patients: Technique and Results, with 3-60 month follow-up. Obes Surg 2000; 10: 233.

71. Sugerman HJ et al Diabetes and hypertension in severe obesity and effects of gastric bypass-induced weight loss. Ann Surg 2003 237: 751-756.

72. Rasheid $\mathrm{S}$ et al. Bypass is an Effective Treatment for Obstructive Sleep Apnea in Patients with Clinically Significant Obesity. OBES SURG 2003; 13: 5.

73. Courcoulas AP et al. Long-term Outcomes of Bariatric Surgery: A National Institutes of Health Symposium. JAMA Surg. 2014; 149 (12): 1323-1329.

74. Arterburn DE et al. Association Between Bariatric Surgery and Long-term Survival. JAMA 2015; 313(1): 62-70.
75. Sjöström L et al. Effects of bariatric surgery on mortality in Swedish obese subjects. N Engl J Med 2007; 357: 741-752.

76. Adams TD et al. (2007) Long-term mortality after gastric bypass surgery. N Engl J Med 357: 753-761

77. Shubeck $S$ et al. Long-term Outcomes Following Bariatric Surgery. JAMA 2018; 319(3): 302-303.

78. Faerber G. Adipositas und chronische Inflammation bei phlebologischen und lymphologischen Erkrankungen. Phlebologie 2018; 47: 55-65.

79. Lim EL et al. Reversal of type 2 diabetes: normalisation of beta cell function in association with decreases pancreas and liver triacylglycerol. Diabetologica 2011; 54: 2506-2514.

80. Steven S, Taylor R. Restoring hyperglycaemia by very low calorie diet in long and short term diabetes. Diabet Med 2015; 32(9): 1149-1155.

81. Stunkard A. The Results of Treatment for Obesity. A Review of the Literature and Report of a Series. AMA Arch Intern Med 1959; 103(1): 79-85.

82. Bennett W, Gurin J. The Dieter's Dilemma: Why Diets Are Obsolete-the New Setpoint Theory of Weight Control. New York: Basic Books 1982.

83. Cogan J Rothblum E. Outcomes of weight-loss Programms. Genetic, Social and General Psychology Monographs 1993; 118 (4).

84. Perri MG et al. Sucess and failure in the treatment of obesity: Where do we go from here? Medicine, Exercise, Nutrition and Health 1995; 4: 255-272.

85. Hensrud DD, Weinsier RL. A prospective study of weight maintenance in obese subjects reduced to normal body weight without weight-loss training American clinical Nutrtion 1994; 60(5): 688-694.

86. Mann T, Tomiyama AJ et al. Medicare's search for effective obesity treatments: diets are not the answer. Am Psychol 2007; 62(3): 220-233.
87. Nordmann A et al. Effects of low carb vs low fat diets on weight loss and cardiovascular risk factors: a meta analysis of randomized controlled trials. Arch Internal Med 2006; 166 (8).

88. Fildes A, Charlton J. Probability of an obese person attaining normal body weight. American Journal of Public Health 2015.

89. Dulloo AG et al. How dieting makes the lean fatter. Obesity reviews 2015; 16 (S1): 25-35.

90. Lowe MR. Dieting: Proxy or Cause of Furture weight gain? Obesity Reviews 2015; 16 (S1): 19-24.

91. Pietiläinen $\mathrm{KH}$ et al. Does dieting make you fat? A Twin study. International Journal of Obesity 2012; 36 (3): 456-464.

92. Tindle HA, Omalu B, Courcoulas A. Risk of suicide after long-term follow-up from bariatric surgery. Am J Med 2010; 123: 1036-1042.

93. Peterhänsel C, Petroff D, Klinitzke G, Kersting A, Wagner B. Risk of completed suicide after bariatric surgery: a systematic review. Obes Rev 2013 May; 14(5): 369-382. doi: 10.1111/obr.12014. Epub 2013 Jan 9 .

94. Baumeister H, Härter M. Mental disorders in patients with obesity in comparison with healthy probands. Int J Obesity 2007; 31(7): 1155-1164.

95. Müller A, Claes L, Smits D, Schag K, de Zwaan M. Lifetime Self-Harm Behaviors Are Not More Prevalent in Bariatric Surgery Candidates than in Community Controls with Obesity. Obes Facts 2018; 11: 109-115.

96. Bild abrufbar unter: https://wellroundedmama. blogspot.com/2015/07/lipedema-treatmentpart-5b-weight-and.html

\section{Kommentar zum Beitrag Bertsch T, Erbacher G. Lipödem - Mythen und Fakten Teil 3. Phlebologie 2018; 47: 188-197}

Da der Autor mich in diesem Beitrag missverständlich zitiert, erfordert dies eine Richtigbzw. Klarstellung.

Der Grundtenor des Artikels, die Besserbarkeit der Lipödemsymptomatik durch Gewichtsabnahme und die damit verbundene verbesserte metabolische (und hormonelle!) Situation findet über weite Teile meine $\mathrm{Zu}$ stimmung.

Strittig ist, ob dies allein durch eine bariatrische Operation erreichbar ist.

Im Rahmen einer eigenen Beobachtungsstudie an 100 Patientinnen mit Lipödem oder Lipödem mit sekundärem Lymphödem, die sich in unserem Hause einer Ernährungstherapie unterzogen hatten, gaben fast $83 \%$ der Befragten nach einer durchschnittlichen Nachbeobachtungszeit von drei Jahren und acht Monaten eine Besserung ihrer Be- schwerden an, mit einer Reduktion der Beschwerdeintensität von durchschnittlich 6,5 auf einer Skala von 1-10 auf 2,5 unmittelbar nach Therapieende und auf 3,0 zum Zeitpunkt der Befragung. Beinvolumina bzw. -umfänge nahmen ab und mit ihnen die Häufigkeit bzw. Intensität von Therapiemaßnahmen.

Die langfristige Erfolgsrate (Stabilisierung der Gewichtsreduktion +/- 10 Kilogramm) aller ernährungsmedizinisch behandelten $\mathrm{Pa}$ tienten (über 450) liegt im Übrigen bei ca. $60 \%$. Auch die Vergleichbarkeit der metabolischen Verbesserungen, hier insbesondere der Erreichung nicht insulinresistenter bzw. nichtdiabetischer Stoffwechselparameter, mit denen nach bariatrischen Operationen können wir mit unseren eigenen Ergebnissen bestätigen (siehe Abb. 2, Phlebologie 2/2018, S. 59).
Es kann also nur wiederholt werden, dass „eine langfristige Nachsorge und Betreuung sowohl nach Adipositaschirurgie als auch nach Ernährungstherapie zwingend erforderlich ist, nur dann zufriedenstellende Langzeitergebnisse möglich sind".

Im übrigen stehe ich der bariatrischen Operation zwar kritisch, aber keineswegs völlig ablehnend gegenüber. Ich habe auch nicht "eindringlich davon abgeraten", sondern lediglich Vor- und Nachteile, bekannte Risiken und Nebenwirkungen genannt, die eine sorgfältige Auswahl und Aufklärung der Patienten erforderlich machen. Im Gegensatz zum Autor dieses Beitrags, der die Adipositaschirurgie als einzige erfolgversprechende Methode darstellt, diese auch bei einem BMI unter 35 $\mathrm{kg} / \mathrm{m}^{2}$ ohne Vorliegen eines Diabetes propagiert und alle konservativen Methoden ab- 
lehnt, rate ich keineswegs pauschal von den operativen Optionen ab, sehe aber die Indikation kritischer und berate Patienten, bei denen diese besteht, ergebnisoffen zu beiden Optionen. Allerdings lehnen die meisten auch derjenigen, denen ich, z.B. wegen eines Rückfalls, zur Operation rate, diese ab. Von den bisher bei uns behandelten Patienten haben sich unseres Wissens lediglich zwei danach doch für eine Operation entschieden.

Immer wieder stellen sich Patienten nach einer Magen-Bypass-Operation mit dem Wunsch nach einer ernährungsmedizinischen Betreuung vor, weil sie nicht weiter abnehmen können oder bereits wieder zugenommen haben. Eine von ihnen, die sich vor wenigen Tagen vorstellte, möchte ich abschließend zu Wort kommen lassen. Sie hatte sich 2013 einer Roux-en-Y- Magenbypassoperation unterzogen (Gewicht damals 135 kg, BMI 44,6, kein Diabetes mell.). Danach Gewichtsreduk- tion von 55 KG im ersten Jahr. 2014 Revisionsoperation wegen Oberbauchhernie und massiver Verwachsungen, dabei Verletzung des Dünndarms, erneute Resektion, Wundheilungsstörungen, Nahtdehiszenz. Seit Anfang 2016 Gewichtszunahme von 15-20 kg. 2017 zweite Schwangerschaft, Geburtsgewicht 2100g. Danach weitere Gewichtszunahme auf jetzt 103 kg (BMI 34,0). Rezidivierendes Erbrechen, Oberbauchschmerzen, DumpingSyndrom, Hypocalcämie, Hypalbulinämie. HbA1c 6,0, Insulinresistenz (HOMA-IR 5,6). "Am Anfang habe ich die OP nicht bereut, jetzt schon. Man wird nicht über die Komplikationen aufgeklärt. Man wird auch nur am Bauch operiert, nicht am Kopf. Ich bin zuckersüchtig, trotz Dumping. Ich musste meine Ernährungsgewohnheiten vollständig ändern, hätte das alles auch ohne OP erreichen können, wenn mir jemand dabei geholfen hätte. Dann wäre mir vielleicht viel erspart geblieben."

Richtig ist, wie von Bertsch gefordert, dass bei der Behandlung von morbid Adipösen „alle spezialisierte Berufsgruppen gemeinsam an einem Strang ziehen müssen: Ernährungsmediziner wie Psychotherapeuten, Internisten und Allgemeinmediziner wie Chirurgen." Das kann aber nicht dazu führen, dass Ernährungsmediziner nur noch die Vor- und Nachsorge bariatrisch operierter Patienten übernehmen sollen, ansonsten aber als dubios, gewinnorientiert und unseriös diffamiert werden.

$$
\begin{array}{r}
\text { Dr. med. Gabriele Faerber } \\
\text { Zentrum für Gefäßmedizin } \\
\text { Paul-Dessau-Str. 3e } \\
22761 \text { Hamburg } \\
\text { info@gefaesszentrum.de }
\end{array}
$$

